\title{
Use of a Daily Lung Compliance Evaluation to Track Progress on ECMO: Successful Use in a Patient With Cystic Fibrosis
}

\author{
Samer Abu-Sultaneh ${ }^{\mathrm{a}, \mathrm{c}}$, Shekhar S. Raja, Brian D Benneywortha, \\ A. Ioana Cristea ${ }^{\mathrm{b}}$, Mara E. Nitu ${ }^{\mathrm{a}}$, Mark R. Rigby ${ }^{\mathrm{a}}$
}

\begin{abstract}
Survival rates of extracorporeal membrane oxygenation (ECMO) for pediatric respiratory failure have been improving and are now about $70 \%$. With this, traditional exclusionary criteria for ECMO may be challenged. We hypothesize that an objective evaluation of pulmonary recovery whilst on ECMO may assist in the care of high risk patients, such as those with cystic fibrosis (CF), both to strategize appropriate decannulation and avoid futility. A 19-yearold female with CF developed septic shock and MRSA-associated acute respiratory distress syndrome. After 4 days, her respiratory status deteriorated and was transitioned to veno-venous ECMO. Due to uncertainty of pulmonary recovery and survival, we instituted a "daily lung compliance trial" (DLCT) to objectively assess pulmonary compliance and function. This included increasing ventilatory support from "rest settings" to moderate non-toxic setting and assessing pulmonary pressures and compliances after $30 \mathrm{~min}$. This provided objective data of lung healing. Due in part to this data, the patient was decannulated from ECMO after 11 days and successfully extubated 2 days later. ECMO can be used for CF patients with acute respiratory failure as a bridge to recovery. Using a DLCT can help guide decision making for respiratory ECMO patients with significant co-morbidities.
\end{abstract}

Keywords: Extracorporeal membrane oxygenation; Extracorporeal life support; Cystic fibrosis; Lung compliance; Acute respiratory distress syndrome; Extracorporeal life support organization

Manuscript accepted for publication October 2, 2013

${ }^{a}$ Department of Pediatrics, Section of Pediatric Critical Care Medicine, Indiana University School of Medicine and Riley Hospital for Children at Indiana University Health, Indianapolis, IN, USA

${ }^{b}$ Department of Pediatrics, Section of Pediatric Pulmonary, Indiana University School of Medicine and Riley Hospital for Children at Indiana University Health, Indianapolis, IN, USA

${ }^{\mathrm{c} C}$ Corresponding author: Samer Abu-Sultaneh, Pediatric Critical Care Indiana University School of Medicine and Riley Hospital for Children at Indiana University Health, 705 Riley Hospital Drive, ROC 4905, Indianapolis, Indiana, USA. Email: sultaneh@iu.edu

doi: http://dx.doi.org/10.14740/jmc1511w

\section{Introduction}

The combination of the advances in technology and experience using extracorporeal membrane oxygenation (ECMO) made over the past three decades are now challenging the traditional concepts of patient eligibility for this life-saving technology [1-3]. Such advances include improvements in cannulation techniques, pumps and oxygenator technology, and anticoagulation management. Given these technical advances, the success of ECMO has improved substantially, increasing the survival of pediatric respiratory ECMO from $57 \%$ to $72 \%$ over the past two decades $[4,5]$. The traditional absolute or relative contraindications for ECMO (including congenital or acquired immune deficiencies and underlying respiratory disease) are now challenged. Because of the irreversibility of the underlying condition, traditionally, ECMO may have been denied to patients with cystic fibrosis (CF) with severe, unrelenting, acute respiratory failure and multisystem organ disease.

Improvement of the care of CF patients has led to a growing population of patients with minimal degrees of pulmonary and multi-organ dysfunction. Coupled with the aforementioned advances in ECMO, it is presently unclear if and when patients with CF should be offered ECMO; and, if so, how to assess lung recovery during support.

We report a case of teenage girl with CF who developed septic shock, ARDS and MODS successfully supported to survival with VV-ECMO. We used a simple, yet novel, approach to assess lung recovery while on ECMO which helped determine the trajectory of recovery and appropriateness for decannulation.

\section{Case Report}

A 19-year-old girl with CF and CF-related pancreatic deficiency and CF related diabetes was admitted to our ICU with vomiting, lethargy and increase work of breathing. Soon after admission she was intubated, fluid resuscitated and placed on vasoactive support (dopamine, epinephrine and norepinephrine) for presumed septic shock. She became oliguric 

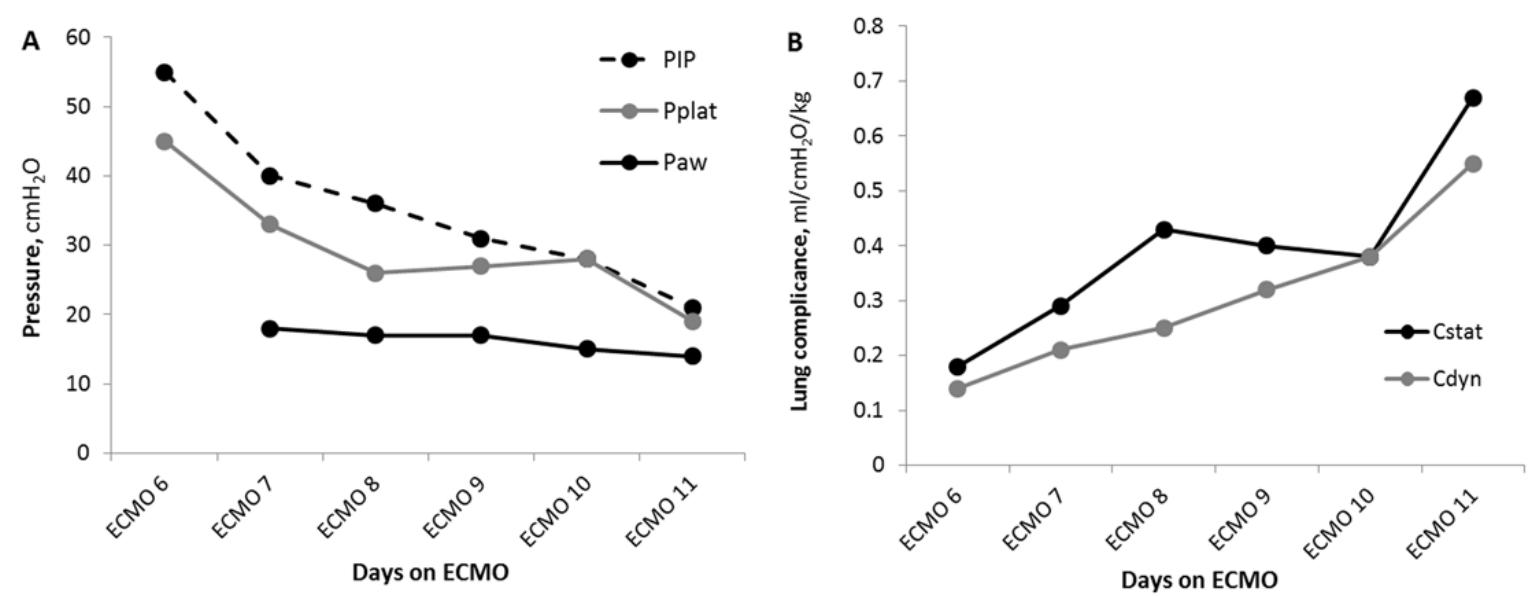

Figure 1. Daily lung compliance trial (DLCT) gives insight to pulmonary improvement on ECMO. Pulmonary pressures (A) and lung compliance (B) during DLCT. Reading (circles) were obtained 30 minutes after initiating DLCT. Cdyn: dynamic compliance; Cstat: static compliance; Paw: mean airway pressure; PEEP: positive end-expiratory pressure; PIP: peak inspiratory pressure; Pplat: plateau pressure.

and her blood urea nitrogen and creatinine became elevated (eventually $43 \mathrm{mg} / \mathrm{dL}$ and $3.9 \mathrm{mg} / \mathrm{dL}$ respectively). She was placed on continuous renal replacement therapy (CRRT) on ICU day 3. Stool PCR was positive for Clostridium difficile and endotracheal tube aspirate culture was positive for methicillin-resistant Staphylococcus aureus (MRSA). Due to worsening respiratory status on ICU day 4 she was placed on high frequency oscillatory ventilation (HFOV) with sig- nificant support $\left(\mathrm{FiO}_{2} 1.0\right.$, mean airway pressure 34, amplitude 98 , and frequency $4 \mathrm{~Hz}$ ). The patient continued to suffer from shock, severe hypoxemia ( $\mathrm{P} / \mathrm{F}$ ratio of 49 and oxygenation index of 63), worsening bilateral lung infiltrates, and a combined respiratory and metabolic acidosis (arterial $\mathrm{pH}$ 7.22, $\mathrm{PaCO}_{2} 61$ with a base deficit of 3 ).

Due to concerns of additional ongoing ventilator associated lung injury, our intensivist and pulmonary care teams

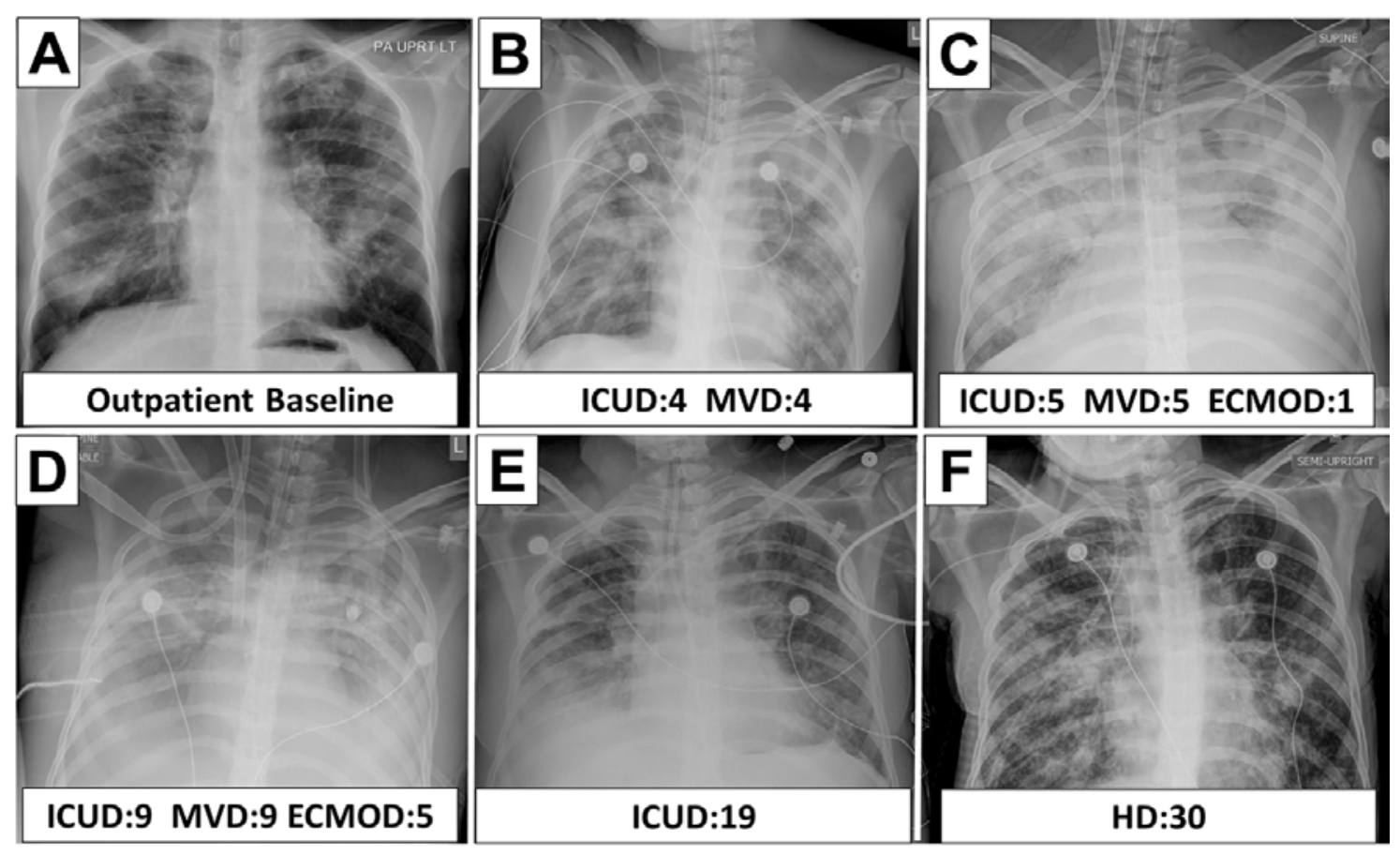

Figure 2. Radiographic course of cystic fibrosis patient while on ECMO. ICUD: ICU day; MVD: mechanical ventilation day; ECMOD: ECMO day; HD: hospital day. 
Table 1. Survival Over Time for CF Patients in ELSO Database by Transplant status*

\begin{tabular}{lll}
\hline & Non-Transplant Related & Transplant Related \\
\hline $1998-2000$ & $1 / 2(50 \%)$ & $4 / 9(44 \%)$ \\
$2001-2003$ & $1 / 3(33 \%)$ & $5 / 10(50 \%)$ \\
$2004-2006$ & $5 / 12(42 \%)$ & $0 / 1(0 \%)$ \\
Mean Survival prior $2007^{\dagger}$ & $7 / 17(41 \%)$ & $9 / 20(45 \%)$ \\
2007 - 2009 & $2 / 8(25 \%)$ & $3 / 3(100 \%)$ \\
2010 - 2012 & $11 / 23(48 \%)$ & $13 / 20(65 \%)$ \\
Mean Survival after $2007^{\dagger}$ & $13 / 31(42 \%)$ & $16 / 23(70 \%)$ \\
\hline
\end{tabular}

$\%$ Reflects the percentage of patients in each year in by transplantation status; *Reflects only first ECMO run of CF patients; †Mean survival is the overall survival during the specified time interval. CF: cystic fibrosis; ELSO: Extracorporeal Life Support Organization; ECMO: extracorporeal membrane oxygenation.

discussed the appropriateness of ECMO. In further review, prior to this acute event, our patient had mild respiratory disease (including $\mathrm{FEV}_{1}$ of $64 \%$, no home oxygen needs) with good medical compliance, only two CF exacerbations the past year, and no neurological issues. Because this seemed like a rare and unconventional indication for ECMO, we conducted a literature review and found only one published case of CF patient that survived ARDS using ECMO without the need for lung transplant [6]. Given her irreversible underling respiratory diagnosis, she might not be considered an optimal ECMO candidate. Yet after family centered multidisciplinary consultation, it was agreed that the potential benefits outweighed the risks. On ICU Day 4 VV ECMO was initiated without complications. She was transitioned to nominal ventilator "rest settings" (SIMV-PC, PIP $=26$, PEEP $=12$, Rate $=8, \mathrm{iT}=1.2, \mathrm{FiO}_{2}=0.5$ ). Over the first few days, the patient continued to require significant ECMO support (namely pump flows of $65-90 \mathrm{~mL} / \mathrm{kg} / \mathrm{min}$, sweep gas at $5-8 \mathrm{~L} / \mathrm{min}$ and $\mathrm{FiO}_{2}$ of 1.0 with post membrane $\mathrm{PaO}_{2}$ around 400).

To aid in pulmonary clearance, serial bronchoscopies were initiated (ECMO day 2, 3 and 5), and nebulized albuterol, acetylcysteine, dornase alfa, hypertonic saline and regular manual hyperinflation were instituted. Intravenous methylprednisolone was started on ECMO day 6. Antibiotic therapy (intravenous vancomycin, metronidazole, piperacillin-tazobactam, tobramycin and enteral vancomycin) was continued.

The approach to weaning ECMO and assessing for decannulation readiness differs between the members of our physician group. As a result, this potentially long ECMO run could have raised several challenges in maintaining the continuity of care. Developing a tool that could objectively assess pulmonary function would be of major importance to care. Thus we developed a serial, standardized approach to regularly document respiratory function and, hopefully, recovery while on ECMO. The "daily lung compliance trial" (DLCT) consists of a temporary increase in the MV settings from the low "rest" to moderate (still non-toxic) settings; SIMV-PRVC with tidal volume of $6 \mathrm{~mL} / \mathrm{kg}$, PEEP 12, PS 10 , rate 12 , iT 1.2 second, $\mathrm{FiO}_{2} 0.5$ for total of 30 minutes. At the end of the DLCT, peak inspiratory pressure (PIP), plateau pressure (Pplat), mean airway pressure (Paw), arterial blood gas, oxygen saturation $\left(\mathrm{SpO}_{2}\right)$, and end tidal $\mathrm{CO}_{2}\left(\mathrm{EtCO}_{2}\right)$ were obtained and oxygenation index (OI), static compliance (Cstat) and dynamic compliance (Cdyn) were calculated.

DLCT was initiated on ECMO day 6. They were well tolerated with arterial $\mathrm{pH} 7.23-7.46, \mathrm{PaO}_{2} 53-69 \mathrm{mmHg}$, $\mathrm{PaCO}_{2} 34-73 \mathrm{mmHg}, \mathrm{SpO}_{2} 85-94 \%$, $\mathrm{EtCO}_{2} 27$ - 52, and OI 11.6 - 16.9. The DLCT proved to be a valuable tool to trend pulmonary improvement. The peak and plateau pressures and lung compliance increases of 3 - 4 fold (Fig. 1) were recorded. This data contributed to our decision to decannulate on ECMO day 11. She was extubated 3 days later (ICU day $15)$ and was discharged home with no supplemental oxygen after 39 days of hospitalization with $\mathrm{FEV}_{1}$ of $46 \%$. Figure 2 shows the radiological course of patient's lung during hospitalization.

\section{Discussion}

ECMO is a rescue therapy for select patients with severe respiratory failure [1-5] that facilitates rest for the lungs and 
Table 2. Survival of Non-Transplant CF Patients Requiring ECMO Based on Clinical Characteristics

\begin{tabular}{|c|c|}
\hline Characteristics & Survival \\
\hline Total non-transplant & $20 / 48(42 \%)$ \\
\hline \multicolumn{2}{|l|}{ Age } \\
\hline$<12$ years & $6 / 14(43 \%)$ \\
\hline $12-17$ years & $5 / 7(71 \%)$ \\
\hline $18-35$ years & $5 / 20(25 \%)$ \\
\hline$>35$ years & $4 / 7(57 \%)$ \\
\hline \multicolumn{2}{|l|}{ Clinical characteristics } \\
\hline vasoactive support & $15 / 33(45 \%)$ \\
\hline HFOV & $6 / 13(46 \%)$ \\
\hline dialysis/renal failure & $3 / 6(50 \%)$ \\
\hline sepsis & $5 / 16(31 \%)$ \\
\hline \multicolumn{2}{|l|}{ Pre-ECMO MV time } \\
\hline$\leq 12$ hours & $0 / 7(0 \%)$ \\
\hline $12-48$ hours & $15 / 20(75 \%)$ \\
\hline$>2$ days & $2 / 13(15 \%)$ \\
\hline \multicolumn{2}{|l|}{ ECMO type } \\
\hline Veno-venous & $14 / 27(52 \%)$ \\
\hline Veno-arterial & $6 / 20(30 \%)$ \\
\hline
\end{tabular}

$\%$ Reflect the percentage of survival in each group; CF: cystic fibrosis; ECMO: extracorporeal membrane oxygenation; MV: mechanical ventilation time; HFOV: high frequency oscillatory ventilation.

recovery by using non-toxic ventilatory settings while providing adequate gas exchange [7]. With improved technology, experience and success, ECMO is increasingly offered to patients with significant comorbidities, some of which might have been considered contraindications in the past [4, 5]. For cystic fibrosis, ECMO has been successfully used as a bridge for lung transplantation in end stage lung disease [8, 14]. Scant data are available regarding the use of ECMO for CF patients without the plan to use this as a bridge to lung transplantation [6]. Patients with pre-existing chronic lung disease are at a greater risk for acquiring additional ventilator associated lung injury. As a result, CF patients (without a transplant plan) may be considered unsuitable candidates for ECMO.

Our query of the ELSO registry found only $91 \mathrm{CF}$ patients supported with ECMO 1998 to mid-2012, with about half of the runs non-transplant related (Table 1). Survival in transplant-related CF ECMO appears to have improved (namely 45 to $70 \%$ before versus after 2007 ) yet the survival rate has not changed for non-transplant related case (about $40 \%$ ) despite an increase in ECMO runs. In assessing the limited data on non-transplant related CF ECMO, it appears that young adulthood (18 - 35 years), sepsis and time to initiate ECMO after starting mechanical ventilation (namely $<12$ hours or $>2$ days) may be risk factors for increased mortality on ECMO (Table 2). It is unclear how inotropic, CVVH, HFOV use or the mode of ECMO (VA versus VV) influences outcome. The length of our patient's ECMO run was consistent with the median duration of ECMO runs for survivors of 10.3 days (IQR 5.6 - 17.9 days) vs. 8 days (IQR 2.9 - 18.1 days) for non-survivals. Due to the underlying disease of our patient, we expected longer run times, yet this data may suggest that if a CF patient will improve on ECMO they will do so within about 1 - 2 weeks. If not, prognosis may be poor. Needless to say, we believe going onto ECMO, our patient met many "high-risk" categories and thus we were warranted 
in our significant concern about her ability to recover.

To obtain objective data, decrease variability between our physicians, and better understand the trajectory of our patient's course, we developed a regular "daily lung compliance trial" to standardize assessment of lung recovery. We intermittently assessed compliance (namely Cstat and Cdyn) on reasonable, and justifiably non-toxic, ventilatory settings, rather than just observing tidal volume over time on very low, and arguably insufficient, "rest settings". DLCT also give a quantitative assessment of gas exchange provided by recovering lungs by obtains blood gases, monitoring $\mathrm{SpO}_{2}$ and $\mathrm{EtCO}_{2}$ while the gas flow through the ECMO oxygenator is completely off. Due to this success, our physician group has integrated DLCTs into standard management of our high-risk respiratory ECMO cases as we feel this will assist in objective evaluation of lung recovery (or lack thereof) within our care team.

\section{Conclusion}

ECMO is a therapeutic modality employed with increased frequency for patients who would have historically been considered sub-optimal candidates. An example is our patient with CF, ARDS, sepsis, inotropic-dependent shock and renal failure. We were able to bridge this patient to recovery using a multifaceted approach, which included a previously unreported way to objectively assess lung recovery while on ECMO. We suggest that using a regular, objective assessment of lung function (namely DLCT) on ECMO can mitigate practice variations and help streamline ECMO weaning, decannulation and prognosis. Furthermore, a standardized assessment tool may aid in improving communication, outcomes and care in challenging ECMO patients.

\section{Conflict of Interest and Role of Funding Source Statements}

The authors declare no scientific, financial or personal conflicts of interest. There was no internal or external funding for this manuscript.

\section{Author Contributions}

Care and decision making of patient: SA, SSR, AIC, MEN, MRR; Data collection and analysis: SA, BDB, MEN, MRR; Drafting and critical editing of manuscript: SA, BDB, MEN, MRR.

\section{References}

1. Davies A, Jones D, Bailey M, Beca J, Bellomo R,
Blackwell N, Forrest P, et al. Extracorporeal Membrane Oxygenation for 2009 Influenza A(H1N1) Acute Respiratory Distress Syndrome. JAMA. 2009;302(17):18881895.

2. Noble DW, Peek GJ. Extracorporeal membrane oxygenation for respiratory failure: past, present and future. Anaesthesia. 2010;65(10):971-974.

3. Brodie D, Bacchetta M. Extracorporeal membrane oxygenation for ARDS in adults. $\mathrm{N}$ Engl $\mathrm{J}$ Med. 2011;365(20):1905-1914.

4. Zabrocki LA, Brogan TV, Statler KD, Poss WB, Rollins MD, Bratton SL. Extracorporeal membrane oxygenation for pediatric respiratory failure: Survival and predictors of mortality. Crit Care Med. 2011;39(2):364-370.

5. Dalton HJ. Extracorporeal life support: moving at the speed of light. Respir Care. 2011;56(9):1445-1453; discuiion 1453-1446.

6. Kuhl T, Langebartels G, Madershahian N, Wahlers T. [Extracorporeal life support given to a 16-year-old girl with cystic fibrosis, candida pneumonia and acute respiratory distress syndrome]. Dtsch Med Wochenschr. 2010;135(42):2071-2075.

7. Peek GJ, Mugford M, Tiruvoipati R, Wilson A, Allen E, Thalanany MM, Hibbert CL, et al. Efficacy and economic assessment of conventional ventilatory support versus extracorporeal membrane oxygenation for severe adult respiratory failure (CESAR): a multicentre randomised controlled trial. Lancet. 2009;374(9698):1351-1363.

8. Aigner C, Wisser W, Taghavi S, Lang G, Jaksch P, Czyzewski D, Klepetko W. Institutional experience with extracorporeal membrane oxygenation in lung transplantation. Eur J Cardiothorac Surg. 2007;31(3):468473; discussion 473-464.

9. Lang G, Taghavi S, Aigner C, Renyi-Vamos F, Jaksch P, Augustin V, Nagayama K, et al. Primary lung transplantation after bridge with extracorporeal membrane oxygenation: a plea for a shift in our paradigms for indications. Transplantation. 2012;93(7):729-736.

10. Boussaud V, Mal H, Trinquart L, Thabut G, DannerBoucher I, Dromer C, Raymond CS, et al. One-year experience with high-emergency lung transplantation in France. Transplantation. 2012;93(10):1058-1063.

11. Nosotti M, Rosso L, Palleschi A, Lissoni A, Crotti S, Marenghi C, Colombo C, et al. Bridge to lung transplantation by venovenous extracorporeal membrane oxygenation: a lesson learned on the first four cases. Transplant Proc. 2010;42(4):1259-1261.

12. Fischer S, Hoeper MM, Tomaszek S, Simon A, Gottlieb J, Welte T, Haverich A, et al. Bridge to lung transplantation with the extracorporeal membrane ventilator Novalung in the veno-venous mode: the initial Hannover experience. ASAIO J. 2007;53(2):168-170.

13. Bermudez CA, Rocha RV, Zaldonis D, Bhama JK, Crespo MM, Shigemura N, Pilewski JM, et al. Extracorpore- 
al membrane oxygenation as a bridge to lung transplant: midterm outcomes. Ann Thorac Surg. 2011;92(4):12261231; discussion 1231-1222.

14. Hayes D, Jr., Kukreja J, Tobias JD, Ballard HO, Hoopes
CW. Ambulatory venovenous extracorporeal respiratory support as a bridge for cystic fibrosis patients to emergent lung transplantation. J Cyst Fibros. 2012;11(1):4045. 